Acta Cryst. (1952). 5, 637

\title{
Interatomic Distances and Atomic Valences in $\mathrm{NaZn}_{13}$ *
}

\author{
By David P. Shommaker†, Richard E. Marsh, Fred J. Ewing and Linus Pauling \\ Gates and Crellin Laboratories of Chemistry, California Institute of Technology, Pasadena 4, California, U.S.A.
}

\author{
(Received 28 January 1952)
}

\begin{abstract}
The crystal structure of $\mathrm{NaZn}_{13}$ and of several homologous compounds $A B_{13}$ was reported by Ketelaar and by Zintl \& Hauke to be based on space group $O_{h}^{6}-F m 3 c$, with $8 \mathrm{Na}$ in $8(a)$ : $\frac{1}{4}, \frac{1}{4}, \frac{1}{4} ; \ldots ; 8 \mathrm{Zn}_{I}$ in $8(b): 0,0,0 ; \ldots ;$ and $96 \mathrm{Zn}_{I I}$ in $96(i): 0, y, z ; \ldots$ Approximate values were reported for the parameters $a_{0}, y$, and $z$; for $\mathrm{NaZn}_{13}$ Zintl \& Hauke reported $12 \cdot 27 \AA, 0 \cdot 178$, and $0 \cdot 122$ for these three parameters. Each $Z_{n}$ is surrounded by twelve $Z_{\text {II }}$ at the vertices of a nearly regular icosahedron, and each $\mathrm{Na}$ by twenty-four $\mathrm{ZnII}$ at the vertices of a snub cube. Our interest in the structure was largely concerned with the valences of the two different kinds of $\mathrm{Zn}$ atoms, it being presumptive that $\mathrm{Zn}_{\mathrm{I}}$ has a larger valence than $\mathrm{Zn}_{\mathrm{Ir}}$ because its icosahedral coordination requires it to be smaller than $\mathrm{Zn}_{\text {II }}$. Lines on new powder photographs of $\mathrm{NaZn}_{13}$ were measured and the intensities were estimated visually with as much precision as possible. Least-squares treatments were employed in order to obtain the best possible values for the three parameters; the values obtained are $a_{0}=12 \cdot 2836 \pm 0 \cdot 0003 \AA, y=0 \cdot 1806 \pm 0 \cdot 0003$, and $z=0 \cdot 1192 \pm 0 \cdot 0003$. The uncertainties given are calculated standard deviations. Analysis of the interatomic distances yields a selfconsistent interpretation in which $\mathrm{Zn}_{\mathrm{I}}$ is assumed to be quinquevalent and $\mathrm{Zn}$ II quadrivalent, while $\mathrm{Na}$ may have a valence of unity or one as high as $1_{4}^{1}$, the excess over unity being suggested by the interatomic distances and being, if real, presumably a consequence of electron transfer. A valence electron number of approximately 432 per unit cell is obtained, which is in good agreement with the value $\mathbf{4 2 8} \cdot 48$ predicted on the basis of a filled Brillouin polyhedron defined by the forms $\{444\},\{640\}$, and $\{800\}$.
\end{abstract}

\section{Introduction}

Some years ago Ketelaar (1937) reported a tentative structure for $\mathrm{NaZn}_{13}$ and five other $A B_{13}$ compounds $\left(\mathrm{KZn}_{13}, \mathrm{KCd}_{13}, \mathrm{CaZn}_{13}, \mathrm{SrZn}_{13}\right.$, and $\left.\mathrm{BaZn}_{13}\right)$. Zintl \& Hauke (1937) reported the same structure for $\mathrm{NaZn}_{13}$ and two other compounds $\left(\mathrm{KZn}_{13}\right.$ and $\left.\mathrm{KCd}_{13}\right)$, and later (1938) for two more $\left(\operatorname{RbCd}_{13}\right.$ and $\left.\mathrm{CsCd}_{13}\right)$. The structure is based on a face-centered cubic lattice. The space group is $O_{h}^{6}-F m 3 c$, with (e.g. for $\mathrm{NaZn}_{13}$ ) eight $\mathrm{Na}$ in $8(a): \frac{1}{4}, \frac{1}{4}, \frac{1}{4} ; \ldots$; eight $\mathrm{Zn}_{\mathrm{I}}$ in $8(b)$ : $0,0,0 ; \ldots ;$ and ninety-six $\mathrm{Zn}_{\mathrm{II}}$ in $96(i): 0, y, z ; \ldots$ The parameters obtained by these workers are summarized in Table 1.

Zintl \& Hauke cited no limits of error, but Ketelaar cited an uncertainty of $0.005 \AA$ for $a_{0}$ and an uncertainty of 0.003 in $y$ and $z$. From comparisons among the intensities obtained for the five compounds that they investigated, Zintl \& Hauke concluded that the $y$ and $z$ parameters are substantially the same for all five.

One of the features of this structure is that the

* Work done in part under Contract N6onr-24432 between the California Institute of Technology and the Office of Naval Research, and in part under a program of research on metals sponsored by the Carbide and Carbon Chemicals Corporation.

Contribution No. 1656 from the Gates and Crellin Labora. tories.

$\dagger$ Present address: Department of Chemistry, Massachusetts Institute of Technology, Cambridge, Massachusetts, U.S.A.

A C 5
Table 1. Parameters obtained in previous work on compounds of type $A B_{13}$

\begin{tabular}{|c|c|c|c|c|c|c|}
\hline \multirow[t]{2}{*}{ Compound } & \multicolumn{3}{|c|}{ Ketelaar } & \multicolumn{3}{|c|}{ Zintl \& Hauke } \\
\hline & $a_{0}^{*}$ & $y$ & $z$ & $a_{0}{ }^{*}$ & $y$ & $z$ \\
\hline $\mathrm{NaZn}_{13}$ & $12 \cdot 255$ & - & $一$ & $12 \cdot 27$ & $0 \cdot 178$ & $0 \cdot 122$ \\
\hline $\mathrm{KZn}_{13}$ & $12 \cdot 335$ & - & - & $12 \cdot 36$ & - & - \\
\hline $\mathrm{KCd}_{13}$ & $13 \cdot 785$ & $0 \cdot 183$ & 0.117 & $13 \cdot 77$ & - & - \\
\hline $\mathrm{CaZn}_{13}$ & $12 \cdot 13$ & - & - & - & - & - \\
\hline $\mathrm{SrZn}_{13}$ & $12 \cdot 215$ & - & 一 & - & - & - \\
\hline $\mathrm{BaZn}_{13}$ & $12 \cdot 33$ & - & - & - & - & - \\
\hline $\mathrm{RbCd}_{13}$ & 一 & - & - & $13 \cdot 88$ & - & - \\
\hline $\mathrm{CsCd}_{13}$ & - & - & - & $13 \cdot 89$ & - & 一 \\
\hline
\end{tabular}

* Values for $a_{0}$ are apparently given in kX. units; Zintl \& Hauke give $\lambda=1.539$ for $\mathrm{Cu} K \alpha$, and 1.537 and 1.541 for the two components.

$\mathrm{Zn}_{\mathrm{I}}$ (or $\mathrm{Cd}_{\mathrm{I}}$ ) at $0,0,0$ is surrounded by twelve $\mathrm{Zn}_{\text {II }}$ at the vertices of a nearly regular icosahedron. The $\mathrm{Zn}_{\mathrm{I}}-\mathrm{Zn}_{\mathrm{II}}$ distance is accordingly substantially smaller than the average $\mathrm{Zn}_{\mathrm{II}}-\mathrm{Zn}_{\mathrm{II}}$ distance. If the icosahedron were regular the ratio of the radial distance to the edge distance would be 0.951 . The observed radial distances $\left(2.65 \AA\right.$ in $\mathrm{NaZn}_{13}, 2.99 \AA$ in $\left.\mathrm{KCd}_{13}\right)$ are in good agreement with the short distances in elementary zine $(2.660 \AA)$ and cadmium $(2.973 \AA)$, to which have been assigned bond numbers of one-half (Pauling, 1947, 1949). This suggests that the atom at $0,0,0$ forms twelve one-half bonds, and hence has a valence of six rather than the usual valence of 4.5 or 4 .

The present investigation was undertaken in order 
to provide unit-cell and positional parameters and interatomic distances for $\mathrm{NaZn}_{13}$ with sufficient precision for a satisfactory interpretation of this structure in terms of atomic valence and bond num. bers.

\section{Experimental 'method}

Samples of $\mathrm{NaZn}_{13}$ were prepared by heating zinc with a calculated excess of sodium in alundum extraction thimbles contained in a steel bomb sealed with copper gaskets. The bomb was filled with nitrogen or argon before being sealed, and was heated for several hours above the melting point of $\mathrm{NaZn}_{13}$ $\left(557^{\circ} \mathrm{C}\right.$.). The bomb was cooled and opened, and the excess of sodium was removed by extraction with anhydrous liquid ammonia. The materials were kept under dry paraffin oil until used. Samples of $\mathrm{KCd}_{13}$ and $K \mathrm{Kn}_{13}$ were prepared in the same way.

Specimens for powder photography were prepared by grinding the materials in dry paraffin oil and drawing the resulting slurry into thin-walled pyrex capillary tubes about $\frac{1}{4} \mathrm{~mm}$. in diameter. Powder photographs were taken with nickel-filtered $\mathrm{Cu} K \alpha$ radiation in a Straumanis-type Philips powder camera of nominal radius $57 \cdot 30 \mathrm{~mm}$. This camera had been constructed with sufficient precision to preclude eccentricity errors, and indeed analysis of powder data obtained with this camera has shown that eccentricity errors are negligibly small. In the case of $\mathrm{NaZn}_{13}$ each exposure was made with two superimposed Eastman No-Screen Dupli-tized X-ray films in the camera; two exposures were made, with exposure times of 2.50 and $9.25 \mathrm{hr}$. at $20 \mathrm{~mA}$. The ratio of these exposure times is $3 \cdot 7$, which is equal to the assumed film factor for $\mathrm{Cu} K \alpha$ radiation. This value of the film factor, which is the factor by which the intensity of the radiation is decreased on passing normally through a single film, was determined at the Eastman Kodak Company laboratories (H. E. Seemann, private communication). One heavily exposed film, two moderately and substantially identically exposed films, and one weakly exposed film were thus obtained, enabling a check to be made on the film factor, which was found to be $3 \cdot 7$ within experimental error.

The relative positions of the lines on the photographs were determined with use of a steel millimeter scale. The intensities of the lines on the $\mathrm{NaZn}_{13}$ photographs were determined visually, by the multiple-film technique, with the use of no external standards.

\section{Determination of the lattice constant}

Reciprocal spacings $\left(1 / d_{h k l}=q_{h k l}=(2 \sin \theta) / \lambda\right)$ were calculated from the positions of the powder lines, and the lines were indexed on the basis of a face-centered cubic lattice. Analysis of a few lines in the backreflection region gave a preliminary value of 0.081409 $\AA^{-1}$ for $1 / a_{0}$. A refinement of $1 / a_{0}$ was carried out by the method of least squares (Whittaker \& Robinson, 1937), with the introduction of an absorption parameter $K$ in conjunction with the absorption function of Nelson \& Riley (1945). The observational equations were of the form

$$
\begin{gathered}
\left(\sqrt{ } w_{i}\right) \frac{d q_{i}}{d\left(1 / a_{0}\right)} \Delta\left(1 / a_{0}\right)+\left(\bigvee w_{i}\right) q_{i} \cdot \frac{1}{2}\left(\frac{\cos ^{2} \theta_{i}}{\sin \theta_{i}}+\frac{\cos ^{2} \theta_{i}}{\theta_{i}}\right) K \\
=\left(\bigvee w_{i}\right)\left(q_{i}^{\text {obs. }}-q_{i}^{\text {calc. }}\right),
\end{gathered}
$$

where $\Delta\left(1 / a_{0}\right)$ and $K$ are the parameters to be solved for, $q_{i}$ is the reciprocal spacing of the $i$ th line, and $w_{i}$ is the weight assigned to the $i$ th line. The coefficient of $K$ contains, besides $w_{i}$, the absorption function of Nelson \& Riley. The weight $w_{i}$ was taken as

$$
w_{i}=w_{i}^{\prime} / \cos ^{2} \theta_{i},
$$

where $w_{i}^{\prime}$ is an auxiliary weight depending on the physical characteristics of the line and has been given arbitrary values ranging from 1 to 4 . The derivative in equation (1) is

$$
\frac{d q_{i}}{d\left(1 / a_{0}\right)}=V\left(h_{i}^{2}+k_{i}^{2}+l_{i}^{2}\right) .
$$

The two normal equations were obtained in the usual way and solved for the two parameters. The results are

$$
\begin{aligned}
& a_{0}=12.2836 \pm 0.0003 \AA, \\
& K=1.35 \times 10^{-4} \pm 2.34 \times 10^{-4},
\end{aligned}
$$

$\left(\lambda=1.5418 \AA\right.$ for unresolved Cu $K \alpha ; 1.54050$ for $\alpha_{1}$; 1.54434 for $\alpha_{2}$ ) where the uncertainties are standard deviations as determined from the residuals and the coefficients of the normal equations (Whittaker \& Robinson, 1937). The absorption parameter $K$ is positive, as it should be, but the value given is evidently not significant. The precision of the latticeparameter determination corresponds to one part in forty thousand. This precision is higher than needed for our purpose, and is not especially significant, as the ambient temperature during photography is not precisely known, the photograph having been taken at room temperature (about $25^{\circ} \mathrm{C}$.). The high precision that is obtainable with this experimental and computational technique justifies accurate knowledge of the temperature at which the exposure is made.

In Table 2 there are given data pertinent to the lattice-parameter determination, as well as intensity data used in the determination of atomic positional parameters.

\section{Determination of atomic positional parameters}

The parameters reported by Zintl \& Hauke were taken as the starting point of the parameter determination. Using these parameters, structure factors were calculated for all of the planes in the sphere of reflection. The atomic form factors of James \& Brindley (1935) were used. (Subsequent calculations made with two 
Table 2. Reciprocal spacings and intensity data for $\mathrm{NaZn}_{13}$

\begin{tabular}{|c|c|c|c|c|c|c|c|c|}
\hline$h k l$ & $w^{\prime}$ & $q_{\text {obs. }}$ & $\begin{array}{c}\Delta q \\
\left(\times 10^{4}\right)\end{array}$ & $w^{\prime \prime}$ & $F_{\text {calc. }}$ & $\underset{\left(\times 10^{-2}\right)}{G_{\text {calc. }}^{2}}$ & $\begin{array}{c}G_{\text {obs. }}^{2} \\
\left(\times 10^{-2}\right)\end{array}$ & $\begin{array}{c}\Delta G^{2} \\
\left(\times 10^{-2}\right)\end{array}$ \\
\hline 200 & - & - & - & 4 & 66.5 & 259 & 236 & -23 \\
\hline 220 & - & - & - & 4 & $-30 \cdot 2$ & 104 & 104 & 0 \\
\hline 222 & - & - & - & - & $2 \cdot 1$ & $\begin{array}{r}0 \\
0\end{array}$ & $<7$ & - \\
\hline 400 & - & - & - & 4 & $18 \cdot 4$ & 18 & 18 & 0 \\
\hline 420 & - & - & - & 4 & $-39 \cdot 6$ & 335 & 307 & -28 \\
\hline 422 & - & - & - & 4 & $90 \cdot 3$ & 1703 & 1502 & -201 \\
\hline 440 & - & - & - & 4 & -60.8 & 370 & 357 & -13 \\
\hline 531 & - & - & - & 4 & $-124 \cdot 2$ & 6074 & 5388 & -686 \\
\hline$\left\{\begin{array}{l}600 \\
442\end{array}\right\}$ & - & - & - & 4 & $\left\{\begin{array}{r}165 \cdot 5 \\
87 \cdot 4\end{array}\right\}$ & 2834 & 2243 & -591 \\
\hline 620 & - & - & - & 4 & $43 \cdot 3$ & 361 & 356 & -5 \\
\hline 622 & - & - & - & 4 & 27.8 & 144 & 140 & -4 \\
\hline 444 & - & - & - & 4 & $74 \cdot 0$ & 332 & 341 & \\
\hline 640 & - & - & - & 4 & $-43 \cdot 3$ & 335 & 330 & -5 \\
\hline 642 & - & - & - & 4 & $28 \cdot 9$ & 293 & 260 & -33 \\
\hline 731 & - & - & - & 4 & $15 \cdot 7$ & 84 & 119 & 35 \\
\hline 800 & - & - & - & 4 & $111 \cdot 0$ & 513 & 556 & 43 \\
\hline$\left\{\begin{array}{l}820 \\
644\end{array}\right\}$ & - & - & - & 4 & $\left\{\begin{array}{l}-36 \cdot 9 \\
-39 \cdot 4\end{array}\right\}$ & 474 & 597 & 123 \\
\hline$\left\{\begin{array}{l}822 \\
660\end{array}\right\}$ & - & - & - & 4 & $\left\{\begin{array}{r}-31 \cdot 0 \\
63.5\end{array}\right\}$ & 474 & 560 & 86 \\
\hline 751 & - & - & - & 4 & $34 \cdot 8$ & 378 & 351 & -27 \\
\hline 662 & - & 一 & - & - & $13 \cdot 6$ & 30 & $<56$ & - \\
\hline 840 & - & - & - & - & $11 \cdot 6$ & 20 & $<60$ & - \\
\hline 753 & - & - & - & 4 & $-83 \cdot 8$ & 2106 & 2153 & 47 \\
\hline 842 & - & - & - & 1 & $39 \cdot 0$ & 452 & 350 & -102 \\
\hline 664 & - & - & - & 4 & $-50 \cdot 1$ & 365 & 369 & 4 \\
\hline 931 & - & - & - & 4 & 65.5 & 1232 & 1282 & 50 \\
\hline 844 & - & 一 & - & 4 & 93.8 & 1227 & 1192 & -35 \\
\hline$\left\{\begin{array}{r}10,0,0 \\
860\end{array}\right\}$ & - & - & - & 4 & $\left\{\begin{array}{r}135.4 \\
73.5\end{array}\right\}$ & 1359 & 1412 & 53 \\
\hline$\left\{\begin{array}{r}10,2,0 \\
862\end{array}\right\}$ & - & - & - & 4 & $\left\{\begin{array}{l}21 \cdot 8 \\
44 \cdot 1\end{array}\right\}$ & 584 & 571 & -13 \\
\hline 951 & - & - & - & 4 & 60.5 & 956 & 1122 & 166 \\
\hline$\left\{\begin{array}{r}10,2,2 \\
666\end{array}\right\}$ & - & - & - & - & $\left\{\begin{array}{r}-6 \cdot 4 \\
-28 \cdot 7\end{array}\right\}$ & 41 & $<107$ & - \\
\hline$\left\{\begin{array}{r}953 \\
10,4,0 \\
864\end{array}\right\}$ & - & - & - & 1 & $\left\{\begin{array}{r}-96.9 \\
-18.8 \\
45.5\end{array}\right\}$ & 2909 & $22 \theta 3$ & -616 \\
\hline $10,4,2$ & - & - & - & 4 & $\begin{array}{r}45.5) \\
24 \cdot 0\end{array}$ & 140 & 79 & -61 \\
\hline 880 & - & 一 & - & 4 & $-37 \cdot 1$ & 79 & 120 & 41 \\
\hline$\left\{\begin{array}{r}11,3,1 \\
971\end{array}\right\}$ & - & - & - & 2 & $\left\{\begin{array}{r}-78 \cdot 2 \\
11.5\end{array}\right\}$ & 1428 & 1282 & -146 \\
\hline$\left\{\begin{array}{r}10,4,4 \\
882\end{array}\right\}$ & - & - & - & 2 & $\left\{\begin{array}{r}-4 \cdot 6 \\
-95 \cdot 6\end{array}\right\}$ & 1037 & 1132 & 95 \\
\hline$\left\{\begin{array}{r}10,6,0 \\
866\end{array}\right\}$ & - & - & - & 4 & $\left\{\begin{array}{c}75 \cdot 4 \\
79 \cdot 0\end{array}\right\}$ & 1325 & 1302 & -23 \\
\hline$\left\{\begin{array}{r}973 \\
10,6,2\}\end{array}\right\}$ & - & - & - & 1 & $\left\{\begin{array}{r}-34 \cdot 4 \\
18 \cdot 6\end{array}\right\}$ & 332 & 314 & -18 \\
\hline $\left.\begin{array}{r}12,0,0 \\
884\end{array}\right\}$ & - & - & - & 4 & $\left\{\begin{array}{c}60.8 \\
13.0\end{array}\right\}$ & 116 & 110 & -6 \\
\hline $11,5,1]$ & - & - & - & 4 & $\begin{array}{r}{[13 \cdot 0]} \\
-14.9\end{array}$ & 46 & 110 & 64 \\
\hline $12,2,0$ & - & - & - & - & -0.9 & 0 & $<95$ & - \\
\hline$\left\{\begin{array}{l}12,2,2 \\
10,6,4\end{array}\right\}$ & - & - & - & 4 & $\left\{\begin{array}{r}58 \cdot 4 \\
-10 \cdot 4\end{array}\right\}$ & 368 & 355 & -13 \\
\hline$\left\{\begin{array}{r}11,5,3 \\
975\end{array}\right\}$ & 1 & 1.0139 & $3 \cdot 6$ & 4 & $\left\{\begin{array}{l}-10.8 \\
-26.8 \\
-14.8\end{array}\right\}$ & 184 & 185 & I \\
\hline
\end{tabular}


Table 2 (cont.)

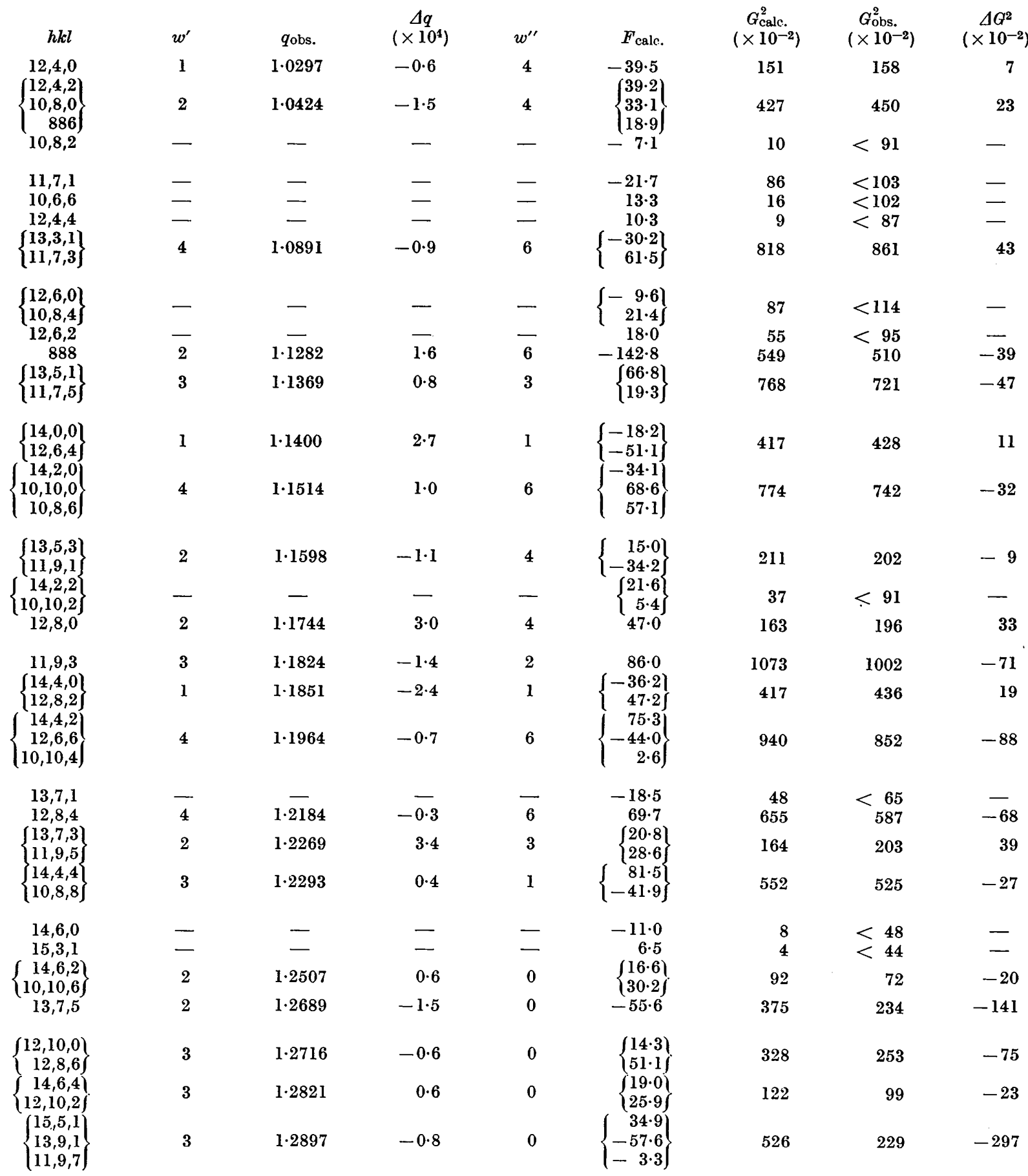

electrons subtracted from the form factor for zinc at all angles to take account of the tight binding of $K$-shell electrons with $\mathrm{Cu} K \alpha$ radiation changed significantly only the scale and temperature parameters.) The structure factors were calculated on the basis of one-eighth of a unit cell. Structure-factor derivatives were also calculated, and a least-squares treatment was carried out, giving $y=0 \cdot 1797, z=0 \cdot 1193$.
However, an error was made in the application of the temperature factor, which resulted in incorrect weights; therefore the structure factors and their derivatives were recalculated on the basis of these parameters and a second least-squares treatment was carried out as described below.

Since many of the powder lines are incapable of resolution into their component reflections, a least- 
squares refinement with respect to the structure factors themselves is inappropriate. The refinement was therefore carried out with respect to quantities $G^{2}$, defined as follows:

$$
\begin{gathered}
G_{i}^{2}(\text { calc. })=t_{i}^{2} \sum_{j} m_{j}\left(F_{j}^{\text {calc. }}\right)^{2}, \\
\left.G_{i}^{2} \text { (obs. }\right)=I_{i}^{\text {obs. }} / k L P \\
\Delta G_{i}^{2}=G_{i}^{2}(\text { obs. })-G_{i}^{2}(\text { calc. })
\end{gathered}
$$

where

$$
t_{i}=\exp \left[-B\left(\sin \theta_{i} / \lambda\right)^{2}\right]
$$

is the temperature factor, $m_{j}$ is the multiplicity of the $j$ th form present in the line, $L$ and $P$ are the Lorentz and polarization factors, and $k$ is a scale factor. The observational equations were of the form

$$
\left(\bigvee w_{i}\right) \frac{\partial G_{i}^{2} \text { (calc.) }}{\partial y} \Delta y+\left(\bigvee w_{i}\right) \frac{\left.\partial G_{i}^{2} \text { (calc. }\right)}{\partial z} \Delta z=\left(\sqrt[V]{ } w_{i}\right) \Delta G_{i}^{2}
$$

for each observed line $i$, where $w_{i}$ is the weight of the measurement of intensity for line $i$ and is defined as follows:

$$
\left.\begin{array}{l}
w_{i}=\frac{w_{i}^{\prime \prime}}{\left(G^{2}(\mathrm{obs} .)\right)^{2}}, G_{i}^{2}(\mathrm{obs} .) \geq 4 G_{\min }^{2} . \\
w_{i}=\frac{w_{i}^{\prime \prime}}{4 G_{i}^{2}(\mathrm{obs} .) G_{\min }^{2}}, 4 G_{\min .}^{2} \geq G_{i}^{2}(\mathrm{obs} .) \geq G_{\min .}^{2}
\end{array}\right\}
$$

where $G_{\mathrm{min}}^{2}$. is the value of $I / k L P$ for the weakest observable line in the neighborhood of the line in question and $w^{\prime \prime}$, like $w^{\prime}$, is an auxiliary weight depending as objectively as possible on physical characteristics of the line which affect the ease of estimating its intensity. For a normal line a value of 4 was assigned to $w^{\prime \prime}$. A line which was well resolved into its two components was given a $w^{\prime \prime}$ not of 8 , but rather of 6 in consideration of the increased difficulty of estimating each component. Values of 1 or 2 were assigned to poorly resolved lines. The leastsquares procedure and the procedure of assigning weights are similar to those of Hughes (1941), except that in the weighting procedure of Hughes equations (9) would contain the number 16 rather than the number 4 .

The temperature-factor parameter $B$ and the scale factor $k$ were determined by a least-squares procedure, with observational equations set up in logarithmic form and with weights obtained from those in equation (9) by multiplying by $\left(G_{i}^{2}\right.$ (obs. $\left.)\right)^{2}$. Since a semilogarithmic plot of $G_{i}^{2}$ (obs.) $/ G_{i}^{2}$ (calc.) against $B$ showed a pronounced deviation from linearity for the last five lines, these lines were omitted from the subsequent treatments. They were much broader than the others, and apparently their intensities were underestimated. The temperature-factor parameter $B$ was found by this treatment to have the value $1.47 \AA^{2}$.

Fifty-two observational equations were set up and reduced in the usual way to two normal equations, which were solved for $\Delta y$ and $\Delta z$. The resulting final values of the positional parameters $y$ and $z$ are

$$
\begin{aligned}
& y=0 \cdot 18063 \pm 0.00025, \\
& z=0 \cdot 11924 \pm 0.00028 .
\end{aligned}
$$

The uncertainties are standard deviations, calculated in the appropriate manner (Whittaker \& Robinson, 1937).

The values of $G_{i}^{2}$ (calc.), calculated with the final parameters, are given in Table 2, where they are compared with $G_{i}^{2}$ (obs.). Considering that the intensities were estimated visually from photographs, the agreement is excellent. The conventional reliability factor $R$, as applied here to the $G^{2}$ rather than to structure factors, i.e.

$$
R=\frac{\sum_{i}\left|\Delta G_{i}^{2}\right|}{\sum_{i} G_{i}^{2} \text { (obs.) }},
$$

calculated for all observable lines except the last five, has a value of $0 \cdot 108$. This corresponds to a value of about 0.05 in the structure factors; the value of $R$ calculated from the structure factors for the twenty. nine non-degenerate lines was found to be 0.050 . The excellence of agreement is presumably due to two main causes: (1) the atoms in the crystals are nearly all of the same kind, so that form-factor errors can be largely compensated for by adjusting the temperature factor; and (2) the visual estimation of the integrated intensity of a line is probably capable of greater precision than the visual estimation of the integrated intensity of a spot obtained on a single-crystal photograph. In single-crystal work, with visual estimation

\begin{tabular}{|c|c|c|c|}
\hline $\begin{array}{l}\text { A. Bonds involving } \mathrm{Zn}_{\mathrm{I}}(0,0,0) \\
\quad \mathrm{Zn}_{I \mathrm{I}}(0, y, z)\end{array}$ & 12 & $2 \cdot 659$ & 0.003 \\
\hline B. Bonds involving $\mathrm{Zn}_{\mathrm{II}}(0, y, z)$ & $\therefore$ & 0.571 & \\
\hline $\begin{array}{l}\operatorname{Zn}_{I I}\left(0, z, \frac{1}{2}-y\right) \\
\operatorname{Zn}_{I}(0,0,0)\end{array}$ & I & $2 \cdot 659$ & \\
\hline $\operatorname{Zn}_{I I}\left(z, \frac{1}{2}-y, 0\right)$ & 2 & $2 \cdot 682$ & 0.0 \\
\hline $\operatorname{Zn}_{\text {II }}(z, 0, y)$ & 4 & $2 \cdot 764$ & 0.0 \\
\hline $\mathrm{Zn}_{I I}(0, y, \bar{z})$ & I & $2 \cdot 929$ & $0 \cdot 0$ \\
\hline $\mathrm{Na}\left(\frac{1}{4}, \frac{1}{4}, \frac{1}{4}\right)$ & 2 & 3.569 & 0.0 \\
\hline $\begin{array}{l}\text { C. Bonds involving } \mathrm{Na}\left(\frac{1}{4}, \frac{1}{4}, \frac{1}{4}\right) \\
\quad \mathrm{Zn}_{\mathrm{II}}(0, y, z)\end{array}$ & 24 & $3 \cdot 569$ & 002 \\
\hline
\end{tabular}
of intensities, $R$ as calculated from the structure factors is seldom less than 0.10 or 0.12 .

The distances between adjacent atoms are given in Table 3. The functional dependence of the distance on the two parameters was taken properly into account in calculating the standard deviations of these distances.

\section{Table 3. Bond distances in $\mathrm{NaZn}_{13}$}

Bond to atom $\quad \begin{aligned} & \text { Number } \\ & \text { of bonds }\end{aligned} \quad D(\AA) \quad \sigma(\AA)$

\section{Discussion of the structure}

Part of the structure, showing the environments of the $\mathrm{Na}$ and $\mathrm{Zn}_{\mathrm{I}}$ atoms, is shown in Fig. 1, and the 
environment of a $\mathrm{Zn}_{\text {II }}$ atom is shown in Fig. 3. The $\mathrm{Na}$ and $\mathrm{Zn}_{\mathrm{I}}$ atoms, taken by themselves, are in the cesium chloride arrangement. They are surrounded by $\mathrm{Zn}_{\text {II }}$ atoms, which form nearly regular snub cubes

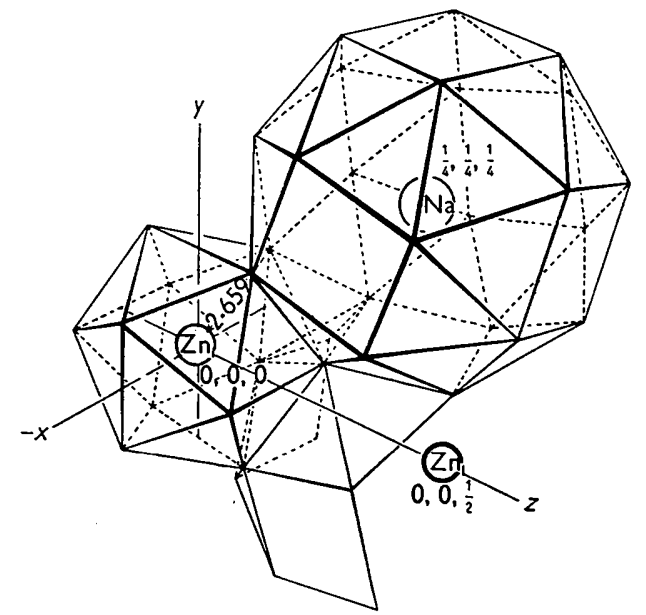

Fig. 1. Drawing of part of the $\mathrm{NaZn}_{13}$ structure, showing the environment of atoms $\mathrm{Zn}_{I}$ and $\mathrm{Na}$, and showing the $\mathrm{Zn}_{\mathrm{I}}-\mathbf{Z n}_{\mathrm{n}}$ distance.

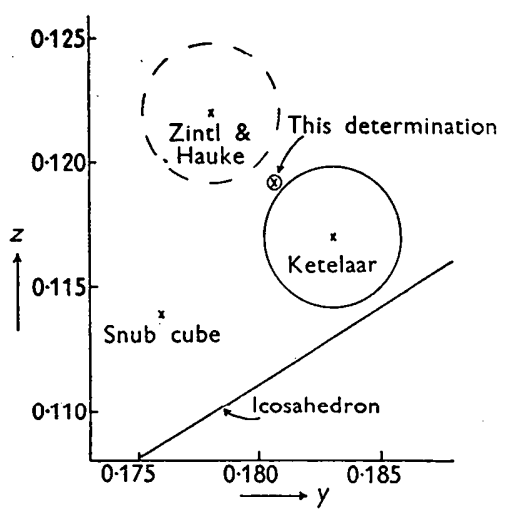

Fig. 2. Parameter plot for $\mathrm{NaZn}_{13}$, showing the line corresponding to a regular icosahedron, the point corresponding to a 'regular' snub cube, and the parameters actually obtained in the previous and present investigations.

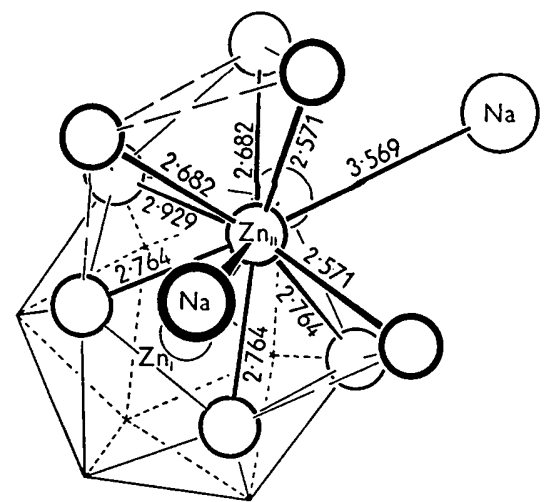

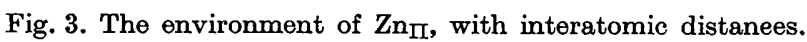
For the $\mathbf{Z n}_{\mathrm{I}}-\mathbf{Z n}_{\mathrm{n}}$ distance, see Fig. 1 . around the $\mathrm{Na}$ atoms and nearly regular icosahedra around the $\mathrm{Zn}_{\mathrm{I}}$ atoms. Each icosahedron is surrounded cubically by, and shares triangular faces with, eight snub cubes, four of which (tetrahedrally arranged about the icosahedron) are right-handed and four (in the opposite tetrahedron) are left-handed. Each snub cube shares triangular faces with eight icosahedra, which are all identical but which alternate in orientation. Between each pair of adjacent icosahedra there is a tetrahedron of $\mathrm{Zn}_{\text {II }}$ atoms, which shares an edge with each of the two icosahedra. Each right-handed snub cube is surrounded octahedrally by six left-handed snub cubes, with which it shares square faces.

For a regular icosahedron $y$ and $z$ are constrained by the relation

$$
y=\frac{1}{2}(1+\sqrt{ } 5) z=1 \cdot 618 z .
$$

A regular snub cube (i.e. one with all edges equal) requires the unique values

$$
y=0 \cdot 1761, z=0 \cdot 1141,
$$

obtained by solution of the equations

$$
z=\frac{\frac{1}{4}-y}{1-2 y}=y-2 y^{2},
$$

which are incompatible with the condition (11) for a regular icosahedron. Hence there exists no set of parameters $y$ and $z$ for which both the icosahedron and the snub cube are regular. For general values of $y$ and $z$ each icosahedron has edges of two different lengths, six of which, of one length, are bisected by twofold axes of the structure, while the remaining twenty-four, of the other length, bound the eight triangular faces which are shared with snub cubes; each snub cube has edges of three different lengths, twenty-four of one length bounding the six square faces, twenty-four of another length bounding the eight triangular faces shared with the icosahedra, and twelve of the third length.

In Fig. 2 conditions (11) and (12) are plotted on a parameter map together with the parameter values reported for $\mathrm{NaZn}_{13}$ by Zintl \& Hauke, those reported for $\mathrm{KCd}_{13}$ by Ketelaar, and those determined for $\mathrm{NaZn}_{13}$ in the present investigation. The uncertainties are indicated by the radii of circles drawn around the points determined by the parameter values; since Zintl \& Hauke reported no uncertainty value, the uncertainty reported by Ketelaar $(0.003)$ was assumed. It is seen that the parameter values obtained in the present work lie between those of Zintl \& Hauke and those of Ketelaar, and that they differ considerably from the values required either by a regular icosahedron or by a regular snub cube.

With the parameters found in the present investigation, the distance from $\mathrm{Zn}_{\mathrm{I}}$ to each of the twelve $\mathrm{Zn}_{\text {II }}$ around it is $2 \cdot 659 \AA$. This value is very close to the value $2 \cdot 660 \AA$ found for the six shorter 
ligates around a given zinc atom in the elementary metal. It has been assumed (Pauling, 1949) that these six ligates represent half-bonds, and that the six longer ligates $(2.907 \AA)$ represent one-sixth bonds, the valence of zinc in the element thus being $4 \cdot 0$. It would therefore seem reasonable to assume that in $\mathrm{NaZn}_{13}$ the $\mathrm{Zn}_{\mathrm{I}}-\mathrm{Zn}_{\text {II }}$ bonds are half-bonds and that accordingly the valence of $Z_{\mathrm{I}}$ is $6 \cdot 0$. The single-bond radius that has been assumed for sexivalent zinc, however, is $1.176 \AA$, which is considerably shorter than the value assumed for quadrivalent zinc, 1.229 $\AA$, in consequence of a larger amount of $d$ character of the bond orbitals. This shorter radius is not consistent with the bond number one-half, which is necessary for a valence of six for $\mathrm{Zn}_{\mathrm{I}}$. It is instead found by trial that the interatomic distances are compatible with the valence 5 for $Z n_{\text {I }}$ and 4 for $Z n_{\text {III. The values for the }}$ single-bond radius for zinc atoms with valence 5 and 4 are $1 \cdot 202$ and $1.229 \AA$, respectively (Pauling, 1949). Values of the bond numbers $n$ calculated from the observed interatomic distances by means of the equation

$$
R(n)=R(1)-0 \cdot 600 \log n
$$

are 0.649 for the $Z n_{\Pi}-Z n_{\Pi}$ bonds with length $2.571 \AA$ (2), 0.424 for $2 \cdot 682 \AA(2), 0.309$ for $2.764 \AA$ (4), and $0 \cdot 164$ for $2.929 \AA$ (1), and also 0.417 for the $Z n_{I}-Z_{n}$ bonds, which have length $2.659 \AA$, and 0.053 for the $\mathrm{Zn}_{\text {II }}-\mathrm{Na}$ bonds, with length $3.569 \AA$. The twelve bonds with bond numbers 0.417 lead to the valence $5 \cdot 00$ for $\mathrm{Zn}_{\mathrm{I}}$, exactly the value assumed. The sum of the bond numbers for the twelve bonds formed by the $\mathrm{Zn}_{\mathrm{II}}$ atom is 4.07 , satisfactorily close to the assumed value $4 \cdot 00$. The valence obtained for sodium, $24 \times 0 \cdot 053$. is $\mathbf{1 \cdot 2 7}$. The excess over unity may be only apparent, and indicate a small compression of the bonds, or it may be real, and due to electron transfer (Pauling, 1950).

Hence, the interatomic distances indicate that the $\mathrm{Zn}_{\text {II }}$ atoms are quadrivalent and the $\mathrm{Zn}_{\mathrm{I}}$ atoms are quinquevalent (corresponding to equal resonance between the quadrivalent electron configuration and the sexivalent configuration). The increase in single-bond radius from $Z \mathrm{n}_{\text {I }}$ to $Z \mathrm{Zn}_{\text {II }}$ combines with the decrease in bond number from the $\mathrm{Zn}_{\mathrm{I}}-\mathrm{Zn}_{\mathrm{II}}$ bonds to the $\mathrm{Zn}_{\mathrm{II}}-\mathrm{Zn}_{\mathrm{II}}$ bonds that mark the edges of the icosahedron to produce the characteristic $5 \%$ difference in length between the radius and the edges of the regular icosahedron.

The brittleness of these intermetallic compounds suggests an electronic structure involving a filled Brillouin zone. It was pointed out by Ketelaar (1937) that the strongest reflection, that of form 531, corresponds to a Brillouin polyhedron for which the inscribed sphere has a volume of 217 electrons per unit cube, which agrees well with the value 216 calculated on the assumption that the sodium atom is univalent and the zinc atoms are bivalent; that is, calculated in the usual Hume-Rothery way. It has also been shown (Pauling \& Ewing, 1948) that the number of valence electrons per unit cell obtained by summing the new metallic valences often agrees strikingly well with the number of electrons required to fill completely a Brillouin polyhedron corresponding to some set of forms with large structure factors; good agreement was found for the $\gamma$ alloys, $\beta$ manganese, $\alpha$ manganese, and other structures.

For $\mathrm{NaZn}_{13}$ the polyhedron 531 corresponds to electron number $238 \cdot 2$. When truncated by 600 and 442 , which are also strongly reflecting forms, the electron number becomes $224 \cdot 38$, slightly larger than the volume of the inscribed sphere and the electron number given by the ordinary valences for $\mathrm{NaZn}_{13}$, 216 , but in good agreement with that for $\mathrm{CaZn}_{13}, 224$. The next form giving very strong reflections is 800 . It is truncated by several other forms that reflect strongly, of which 444 is the most important. The polyhedron formed by 800,444 , and 640 has an electron number of 428.48 . The only other moderately strongly reflecting form in this region is 642 , which has, however, only a very slightly truncating action, which would reduce the electron number to 428.02. Both of these polyhedra are satisfactory, in that the ratio of maximum radius to minimum radius is close to unity, being only $1 \cdot 17$; the polyhedron accordingly does not deviate greatly from sphericity. The value 428 for the electron number given by the Brillouin polyhedron is close to that corresponding to the valences reported above; the sum of the valences, assuming 5 for $\mathrm{Zn}_{\mathrm{I}}, 4$ for $\mathrm{Zn}_{\text {II }}$, and 1 for $\mathrm{Na}$, is 432 . Exact filling of the zone would be obtained by taking the valence of $\mathrm{Zn}_{\mathrm{II}}$ as $3 \cdot 94$, instead of $4 \cdot 00$.

\section{Potassium compounds}

Measurements of our powder photographs of $\mathrm{KZn}_{13}$ and $\mathrm{KCd}_{13}$ gave $a_{0}=12 \cdot 360 \AA$ for the former and $a_{0}^{*}=13.803 \AA$ for the latter, in both cases without least-squares refinement, and with a standard deviation of about $0.005 \AA$. These values are in good agreement with those previously reported by Zintl \& Hauke and by Ketelaar. The lattice constant for $\mathrm{KZn}_{13}$ is only $0.6 \%$ larger than that for $\mathrm{NaZn}_{13}$, while that for $\mathrm{KCd}_{13}$ is about $12 \%$ larger than those for the two zinc compounds. The ratio of the lattice constants for $\mathrm{KCd}_{13}$ and $\mathrm{KZn}_{13}$ is in fact $1 \cdot 117$, which is very close to the ratio, $1 \cdot 119$, of the atomic radii of cadmium and zinc for coordination 12. Hence it appears that if the $y$ and $z$ parameters for $\mathrm{KCd}_{13}$ and $\mathrm{KZn}_{13}$ are the same as for $\mathrm{NaZn}_{13}$, as is probably true to a close approximation, the situation regarding bonding and valency is essentially the same for the cadmium atoms in $\mathrm{KCd}_{13}$ and for the zinc atoms in $\mathrm{KZn}_{13}$ as it is for the zinc atoms in $\mathrm{NaZn}_{13}$.

We thank Mr Jack Inman for assistance in preparing the compounds, and Mrs Nan Arp and Miss Linda Pauling for assistance with the calculations. 


\section{References}

Hughes, E. W. (1941). J. Amer. Chem. Soc. 63, 1737.

JAMES, R. W. \& BRIndley, G. W. (1935). International Tables for Crystal Structure Determination, p. 571. Berlin: Borntraeger.

KetelaAa, J. A. A. (1937). J. Chem. Phys. 5, 668.

Nelson, J. B. \& Riley, D. P. (1945). Proc. Phys. Soc. $57,160$.

Pauling, L. (1947). J. Amer. Chem. Soc. 69, 546.
Pauling, L. (1949). Proc. Roy. Soc. A, 196, 343.

Pauling, L. (1950). Proc. Nat. Acad. Sci., Wash. 36, 533.

Pauling, L. \& Ewing, F. (1948). Rev. Mod. Phys. 20, 112.

WhitTAKER, E. T. \& RoBinson, G. (1937). The Calculus of Observation, chap. 9. London: Blackie.

ZiNTL, E. \& Hauke, W. (1937). Nature, Lond. 25, 717.

ZiNTL, E. \& HAUKE, W. (1938). Z. Elektrochem. 44, 104.

Acta Cryst. (1952). 5, 644

\title{
The Crystal Structure of L-Glutamine
}

\author{
By W. Cochran and Bruce R. Penfold* \\ Crystallographic Laboratory, Cavendish Laboratory, Cambridge, England
}

(Received 12 March 1952)

\begin{abstract}
The crystal structure of L-glutamine has been determined by means of electron-density projections on the (001) and (010) planes. The (001) projection was solved by direct sign-determining methods which are described. A direct indication is obtained from a map of $\left(\varrho_{o}-\varrho_{c}\right)$ of the positions of all the hydrogen atoms. Bond lengths have been measured with a standard deviation of $0.024 \AA$ and are compared with those in related structures. A correlation between the double-bond character of the $\mathrm{C}-\mathrm{N}$ bond of the amide group and the reactivity of this group in glutamine and acetamide is pointed out. Cohesion of the crystal is provided by five hydrogen bonds per molecule (the maximum number possible) extending in three dimensions and in each case linking a nitrogen and an oxygen atom.
\end{abstract}

\section{Introduction}

Glutamine, the $\gamma$-amide of glutamic acid, is of great biological importance. It occurs in the free state and as part of the protein structure in plants and animals, and plays an essential part in many metabolic processes. Its physiological roles have been extensively studied (Archibald, 1945). Considered simply as a chemical compound it has unusual properties, and its crystal-structure analysis was undertaken mainly with a view to providing information which might help to throw light on these characteristics. The amide group is uniquely labile, being rapidly hydrolysed in buffered solutions and being decomposed by nitrous acid in conditions under which all other amides are unchanged (Plimmer, 1925; Taylor, 1930). Hydrolysis leads to the formation of the five-membered lactam ring compound pyrrolidone-carboxylic acid. Neither this reaction nor the highly reactive nature of the amide group in general have been accounted for satisfactorily and it is desirable that the configuration and dimensions of the molecule should be determined as accurately as possible before an attempt is made to explain these chemical features. The analysis may also be regarded as a contribution to the more general study of amino-acid structures. The method by which

* Now at Canterbury University College (University of New Zealand), Christchurch C.1, New Zealand. the structure was solved is of considerable interest in itself as no assumptions were made as to the configuration of the molecule. This direct method is described in some detail.

\section{Experimental}

The material used was kindly supplied by Dr R. G. Westall of the Cambridge Low Temperature Research Station. It was in the form of a white powder and contained less than $1 \%$ impurity. Recrystallization from water at a temperature between 0 and $5^{\circ} \mathrm{C}$. produced semitransparent needles up to $1.5 \mathrm{~mm}$. long and with maximum mean diameter of cross section $0.2 \mathrm{~mm}$. Elongation was in the direction of the short crystallographic axis.

\section{Crystallographic and physical data}

L-Glutamine $\mathrm{C}_{5} \mathrm{H}_{10} \mathrm{O}_{4} \mathrm{~N}_{2}$; m.p. $184-185^{\circ}$ C.; orthorhombic,

$$
a=16 \cdot 01, b=7 \cdot 76, c=5 \cdot 10 \AA \text { (all } \pm 0 \cdot 3 \% \text { ). }
$$

Absent spectra: $h 00,0 k 0,00 l$ when $h, k, l$ respectively is odd. Space group $P 2_{1} 2_{1} 2_{1}$. Four molecules per unit cell. Density (calc.) $=1.52$ g.cm. ${ }^{-3}$, density (obs.) $=$ 1.54 g.cm. ${ }^{-3}$. Absorption coefficient for $\mathrm{Cu} K \alpha$ radiation $\mu=12 \cdot 2 \mathrm{~cm} \cdot \cdot^{-1}$.

$\mathrm{Cu} K \alpha$ radiation was used throughout, and complete 